\title{
CLASSIFICATION OF SOLUTIONS TO A CRITICALLY NONLINEAR SYSTEM OF ELLIPTIC EQUATIONS ON EUCLIDEAN HALF-SPACE
}

\author{
MATHEW R. GLUCK AND LEI ZHANG
}

\begin{abstract}
For $N \geq 3$ and non-negative real numbers $a_{i j}$ and $b_{i j}(i, j=1, \cdots, m)$, the semi-linear elliptic system

$$
\left\{\begin{array}{ll}
\Delta u_{i}+\prod_{j=1}^{m} u_{j}^{a_{i j}}=0 & \text { in } \mathbb{R}_{+}^{N} \\
\frac{\partial u_{i}}{\partial y_{N}}=c_{i} \prod_{j=1}^{m} u_{j}^{b_{i j}} & \text { on } \partial \mathbb{R}_{+}^{N}
\end{array} \quad i=1, \cdots, m\right.
$$

is considered, where $\mathbb{R}_{+}^{N}$ is the upper half of $N$-dimensional Euclidean space. Under suitable assumptions on the exponents $a_{i j}$ and $b_{i j}$, a classification theorem for the positive $C^{2}\left(\mathbb{R}_{+}^{N}\right) \cap$ $C^{1}\left(\overline{R_{+}^{N}}\right)$-solutions of this system is proven.
\end{abstract}

\section{INTRODUCTION}

Let $N \geq 3$ be a positive integer and let $\mathbb{R}_{+}^{N}=\left\{\left(y_{1}, \cdots, y_{N}\right) \in \mathbb{R}^{N}: y_{N}>0\right\}$ denote the upper half of $N$-dimensional Euclidean space. Fix a positive integer $m$ and set $J=\{1, \cdots, m\}$. Let $\mathcal{A}=\left[a_{i j}\right]$ be an $m \times m$ matrix with nonnegative entries. We are concerned with the classical solutions of the semi-linear elliptic system

$$
\Delta u_{i}+\prod_{j=1}^{m} u_{j}^{a_{i j}}=0 \quad \text { in } \Omega \subset \mathbb{R}^{N} \text { for all } i \in J .
$$

This system and its variants have been studied extensively in numerous contexts. For example, (1.1) arises as the system of equations for a steady-state solution to the corresponding parabolic reaction-diffusion system. In particular, when $m=2$ the system

$$
\begin{cases}\frac{\partial u_{1}}{\partial t}=\Delta u_{1}+u_{1}^{a_{11}} u_{2}^{a_{12}} & \text { for } y \in \Omega, t>0 \\ \frac{\partial u_{2}}{\partial t}=\Delta u_{2}+u_{1}^{a_{21}} u_{2}^{a_{22}} & \text { for } y \in \Omega, t>0\end{cases}
$$

has received much attention. For example, when $a_{11}=a_{22}=0$ (1.2) gives a simple model for heat propagation in a two-component combustible mixture [11]. Variants of (1.2) have also been used to model the diffusing densities of two biological species when each specie finds its subsidence from the activity of the other specie [16]. It is well-known that a thorough understanding of (1.1) is highly beneficial to obtaining an understanding of (1.2). For example, under appropriate assumptions on $\mathcal{A}$, in [20] and [21] Mitidieri proved nonexistence results for (1.1) when $\Omega=\mathbb{R}^{N}$ and $m=2$. These results were refined by Zheng in [24] and then used to derive blow-up (in time) estimates for solutions of (1.2) that satisfy suitable initial and boundary conditions. For more results concerning these parabolic systems and their variants the reader is referred to [15], [7] and the references therein.

An interesting case of (1.1) arises when $\mathcal{A}$ satisfies

2010 Mathematics Subject Classification. Primary 35J57; Secondary 35J66, 35K57.

Key words and phrases. Nonlinear elliptic systems. 


$$
\begin{cases}a_{i j} \geq 0 & \text { for all }(i, j) \in J \times J \\ \mathcal{A} \text { is irreducible } & \\ \sum_{j=1}^{m} a_{i j}=\frac{N+2}{N-2} & \text { for all } i \in J\end{cases}
$$

Recall that an $m \times m$-matrix $\mathcal{A}$ is called irreducible if there is no partition $J=I_{1} \cup I_{2}$ such that $a_{i j}=0$ for all $i \in I_{1}$, and $j \in I_{2}$. When $m=1$ equations (1.1) reduce to

$$
\Delta u+K u^{(N+2) /(N-2)}=0
$$

with $K=1$. Equation (1.4) has been studied extensively as it arises in relation to the famous Yamabe problem. The Yamabe problem asks whether it is always possible to conformally deform the metric $g$ of a given smooth compact Riemannian manifold to a metric $\hat{g}=u^{4 /(N-2)} g$ whose scalar curvature is constant. Through the works of Trudinger [23], Aubin [1] and Schoen [22], the Yamabe problem was proven affirmative. See [14] and the references therein for results regarding the Yamabe problem. For $\mathcal{A}$ satisfying (1.3) and $\Omega=\mathbb{R}^{N}$, the classical solutions of (1.1) were classified by Chipot, Shafrir and Wolansky in [5] (see also [6]). Their result is the following.

Theorem 1.1 (Chipot, Shafrir and Wolansky [5]). Suppose $\mathcal{A}$ satisfies (1.3). If $u_{1}, \cdots, u_{m}$ are positive $C^{2}\left(\mathbb{R}^{N}\right)$-solutions of (1.1) with $\Omega=\mathbb{R}^{N}$ then

$$
u_{i}(y)=\frac{\beta_{i}}{\left(\sigma^{2}+\left|y-y^{0}\right|^{2}\right)^{(N-2) / 2}} \quad \text { for all } i \in J,
$$

for some $y^{0} \in \mathbb{R}^{N}$ and some positive constants $\sigma^{2}$ and $\beta_{1}, \cdots, \beta_{m}$ satisfying

$$
\log \beta_{i}=\sum_{j=1}^{m} a_{i j} \log \beta_{j}-\log \left(\sigma^{2} N(N-2)\right) \quad \text { for all } i \in J .
$$

This theorem is the system-generalization of the classification of entire solutions to (1.4) given in [3].

Many interesting questions involving variants of (1.4) have been considered. For example, for real numbers $K$ and $c$ the equations

$$
\begin{cases}\Delta u+K u^{(N+2) /(N-2)}=0 & \text { in } \mathbb{R}_{+}^{N} \\ \frac{\partial u}{\partial y_{N}}=c u^{N /(N-2)} & \text { on } \partial \mathbb{R}_{+}^{N}\end{cases}
$$

arise in relation to the boundary-Yamabe problem which seeks to determine whether the metric $g$ of smooth compact Riemannian manifold $M$ with boundary can be conformally deformed into a metric $\hat{g}$ such that both the scalar curvature and the boundary mean curvature of $\hat{g}$ are constant. The boundary-Yamabe problem is still open. For a detailed discussion on the boundary-Yamabe problem, the reader is referred to Escobar [9, 10], Han-Li [12, 13], Marques [19] and the references therein. The solutions of equations (1.7) were classified separately by Li and Zhu in [18] and Chipot, Shafrir and Fila in 4]. Later in [17, the solutions of (1.7) with more general nonlinearities were classified. The result is as follows

Theorem 1.2 (Li-Zhu [18, Chipot-Shafrir-Fila [4] and Li-Zhang [17]). If $u$ is a non-negative $C^{2}\left(\mathbb{R}_{+}^{N}\right) \cap C^{1}\left(\overline{\mathbb{R}_{+}^{N}}\right)$-solution of (1.7) with $K=N(N-2)$, then either $u \equiv 0$ or there exists $\sigma>0$ and $\left(y_{1}^{0}, \cdots, y_{N-1}^{0}\right) \in \partial \mathbb{R}_{+}^{N}$ such that

$$
u(y)=\left(\frac{\sigma}{\sigma^{2}+\left|y-y^{0}\right|^{2}}\right)^{(N-2) / 2} \quad \text { for all } y \in \mathbb{R}_{+}^{N},
$$


where $y^{0}=\left(y_{1}^{0}, \cdots, y_{N-1}^{0}, y_{N}^{0}\right)$ and $y_{N}^{0}=\sigma c /(N-2)$.

In this paper, an analogue of Theorem 1.2 is proven for the generalization of (1.7) to a system of equations. To generalize the boundary nonlinearity in (1.7) let $c_{1}, \cdots, c_{m}$ be real numbers and let $\mathcal{B}=\left[b_{i j}\right]$ be an $m \times m$ matrix satisfying

and consider the system

$$
\begin{cases}b_{i j} \geq 0 & \text { for all }(i, j) \in J \times J \\ \sum_{j=1}^{m} b_{i j}=\frac{N}{N-2} & \text { for all } i \in J \\ b_{i j}=\frac{N}{N-2} \delta_{i j} & \text { for all } i \in J \text { such that } c_{i} \geq 0\end{cases}
$$

$$
\left\{\begin{array}{ll}
\Delta u_{i}+\prod_{j=1}^{m} u_{j}^{a_{i j}}=0 & \text { in } \mathbb{R}_{+}^{N} \\
\frac{\partial u_{i}}{\partial y_{N}}=c_{i} \prod_{j=1}^{m} u_{j}^{b_{i j}} & \text { on } \partial \mathbb{R}_{+}^{N} \\
u_{i}>0 & \text { on } \overline{\mathbb{R}_{+}^{N}}
\end{array} \quad \text { for all } i \in J .\right.
$$

Our main theorem is as follows.

Theorem 1.3. Suppose $\mathcal{A}$ satisfies (1.3) and $\mathcal{B}$ satisfies (1.8). If $\left(u_{1}, \cdots, u_{m}\right)$ is a $C^{2}\left(\mathbb{R}_{+}^{N}\right) \cap$ $C^{1}\left(\overline{\mathbb{R}_{+}^{N}}\right)$-solution of (1.9) then there exist positive constants $\sigma, \beta_{1}, \cdots, \beta_{m}$ satisfying (1.6) and $\left(y_{1}^{0}, \cdots, y_{N-1}^{0}\right) \in$ $\partial \mathbb{R}_{+}^{N}$ such that $u_{i}$ is given by (1.5) with $y^{0}=\left(y_{1}^{0}, \cdots, y_{N-1}^{0}, y_{N}^{0}\right)$, where

$$
y_{N}^{0}=\sigma^{2} N c_{i} \prod_{j=1}^{m} \beta_{j}^{b_{i j}-a_{i j}} \quad \text { for all } i \in J .
$$

Remark 1.4. The third item of (1.8) says that if $i \in J$ is an index for which $c_{i} \geq 0$, then the boundary equation for $u_{i}$ is

$$
\frac{\partial u_{i}}{\partial y_{N}}=c_{i} u_{i}^{N /(N-2)} \quad \text { on } \partial \mathbb{R}_{+}^{N}
$$

This assumption is made for convenience as it makes some of the proofs simpler. See, for example the proof of Claim 3.4 .

The proof of Theorem 1.3 is via the method of moving spheres and is inspired by the proofs of Theorems 1.2 and 1.1 given in [17] and [5] respectively. The organization of this paper is as follows. In Section 2 we show that the moving sphere process can start. In Section 3 we obtain a symmetry relation between $u_{i}$ and its "critical" Kelvin transformations. In Section 4 we first use a calculus lemma to deduce the form of the restriction of $u_{i}$ to $\partial \mathbb{R}_{+}^{N}$. Next we transform the problem defined on $\mathbb{R}_{+}^{N}$ to a new problem defined on a ball. After determining that the solutions of the transformed problem must be radial, a system of ODE is obtained and the solution to this system is determined. The conclusion of Theorem 1.3 will follow after returning to the original problem.

Throughout, $C$ will be used to denote a positive constant depending only on $N$. The value of $C$ may change from line to line.

\section{The Moving Sphere Process Can Start}

Let $u_{1}, \cdots, u_{m}$ be as in the hypotheses of Theorem 1.3. As the proof of Theorem 1.3 is via the method of moving spheres, we wish to consider the following $\partial \mathbb{R}_{+}^{N} \times(0, \infty)$-indexed family of Kelvin inversions of $u_{i}$. For $x \in \partial \mathbb{R}_{+}^{N}$ and $\lambda>0$ let

$$
\Sigma_{x, \lambda}=\mathbb{R}_{+}^{N} \backslash \bar{B}_{\lambda}(x)
$$

and define 


$$
u_{i, x, \lambda}(y)=\left(\frac{\lambda}{|y-x|}\right)^{N-2} u_{i}\left(x+\frac{\lambda^{2}(y-x)}{|y-x|^{2}}\right) \quad \text { for } y \in \overline{\mathbb{R}_{+}^{N}} \backslash\{x\} \text { and } i \in J .
$$

By using (1.3), (1.8) and (1.9) and computing directly, one may verify that $u_{1, x, \lambda}, \cdots, u_{m, x, \lambda}$ satisfy

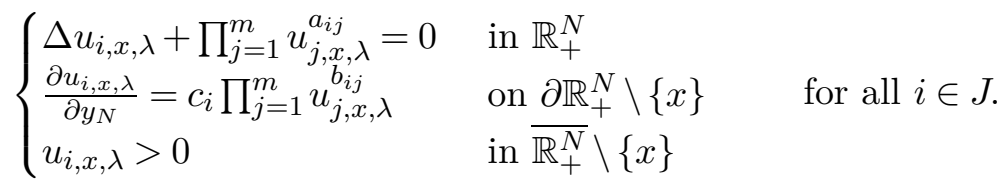

Since we want to compare $u_{i}$ to $u_{i, x, \lambda}$, we define the differences

$$
w_{i, x, \lambda}(y)=u_{i}(y)-u_{i, x, \lambda}(y) \quad \text { for } y \in \overline{\mathbb{R}_{+}^{N}} \backslash\{x\} \text { and } i \in J .
$$

Using (1.9) and (2.1) one can verify that $w_{i, x, \lambda}$ satisfies

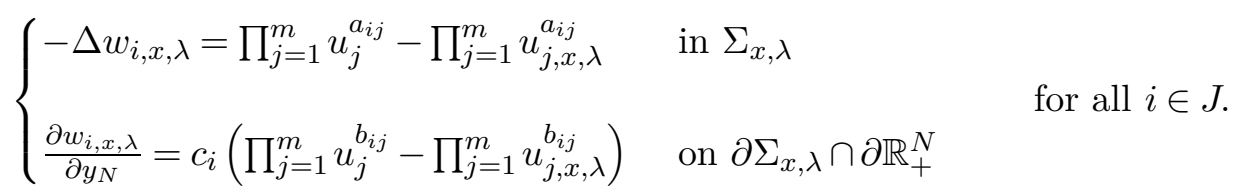

Moreover,

$$
w_{i, x, \lambda}=0 \quad \text { on } \partial \Sigma_{x, \lambda} \cap \partial B_{\lambda}(x) \text { for all } i \in J .
$$

As the proofs of many of the propositions given will be similar for $x=0$ and for general $x \in \partial \mathbb{R}_{+}^{N}$, when considering $x=0$ we will use the following simplified notation

$$
\Sigma_{0, \lambda}=\Sigma_{\lambda}, \quad u_{i, 0, \lambda}=u_{i, \lambda} \quad \text { and } \quad w_{i, 0, \lambda}=w_{i, \lambda} .
$$

Proposition 2.1. For each $x \in \partial \mathbb{R}_{+}^{N}$, there exists $\lambda_{0}(x)>0$ such that for all $\lambda \in\left(0, \lambda_{0}(x)\right)$,

$$
w_{i, x, \lambda} \geq 0 \quad \Sigma_{x, \lambda} \text { for all } i \in J .
$$

According to Proposition [2.1, for $x \in \partial \mathbb{R}_{+}^{N}$, we may define

$$
\bar{\lambda}(x)=\sup \left\{\lambda>0: w_{i, x, \mu} \geq 0 \text { in } \Sigma_{x, \mu} \text { for all } \mu \in(0, \lambda) \text { and all } i \in J\right\} .
$$

For convenience, the proof of Proposition 2.1 will only be given for $x=0$ and the notation in (2.4) will be used. The proof for general $x \in \partial \mathbb{R}_{+}^{N}$ is similar to the proof for $x=0$. We begin by establishing three lemmas.

Lemma 2.2. There exists $r_{0}>0$ such that for all $i \in J$ and all $\lambda \in\left(0, r_{0}\right)$,

$$
w_{i, \lambda}(y)>0 \quad \text { for all } y \in \overline{B_{r_{0}}^{+}} \backslash \bar{B}_{\lambda} .
$$

Proof. For $(r, \theta) \in[0, \infty) \times \overline{\mathbb{S}_{+}^{N-1}}$ and $i \in J$ set $g_{i}(r, \theta)=r^{(N-2) / 2} u_{i}(r, \theta)$, where $\overline{\mathbb{S}_{+}^{N-1}}$ is the closed, $(N-1)$-dimensional upper half sphere. Set

$$
r_{0}=\min \left\{1, \frac{N-2}{4}\left(\min _{j \in J} \frac{\min }{B_{1}^{+}} u_{j}\right)\left(\max _{j \in J}\left\|D u_{j}\right\|_{C^{0}\left(\overline{B_{1}^{+}}\right)}\right)^{-1}\right\} .
$$

For all $0<r \leq r_{0}$ and for all $i \in J$, we have 


$$
\frac{\partial g_{i}}{\partial r}(r, \theta) \geq r^{(N-4) / 2}\left(\frac{N-2}{2} \frac{\min }{B_{1}^{+}} u_{i}-r\left\|D u_{i}\right\|_{C^{0}\left(\overline{B_{1}^{+}}\right)}\right)>0 .
$$

In particular, if $0<\lambda \leq r_{0}$ then with $\theta=y /|y|$,

$$
w_{i, \lambda}(y)=|y|^{(2-N) / 2}\left(g_{i}(|y|, \theta)-g_{i}\left(\frac{\lambda^{2}}{|y|}, \theta\right)\right)>0 \quad \text { for all } y \in \overline{B_{r_{0}}^{+}} \backslash \bar{B}_{\lambda} \text { and all } i \in J
$$

Lemma 2.3. If $i$ is an index for which $c_{i}<0$, then $\liminf _{|y| \rightarrow \infty}|y|^{N-2} u_{i}(y)>0$.

Proof. If $c_{i} \geq 0$ for all $i \in J$, there is nothing to prove. Otherwise, fix $R>0$ and fix $i \in J$ for which $c_{i}<0$. By (2.1) the hypotheses of Lemma 5.1 are satisfied by $u_{i, R}$. Therefore, for each $z \in \overline{B_{R}^{+}} \backslash\{0\}$

$$
\left(\frac{R}{|z|}\right)^{N-2} u_{i}\left(\frac{R^{2} z}{|z|^{2}}\right)=u_{i, R}(z) \geq \min _{\partial B_{R} \cap \mathbb{R}_{+}^{N}} u_{i, R}=\min _{\partial B_{R} \cap \mathbb{R}_{+}^{N}} u_{i} .
$$

Now, if $y \in \overline{\mathbb{R}_{+}^{N}} \backslash B_{R}$, set $z=R^{2} y /|y|^{2}$. Then $z \in \overline{B_{R}^{+}} \backslash\{0\}, y=R^{2} z /|z|^{2}$, and the above inequalities give

$$
u_{i}(y) \geq\left(\min _{\partial B_{R} \cap \overline{\mathbb{R}_{+}^{N}}} u_{i}\right) R^{N-2}|y|^{2-N} .
$$

Lemma 2.3 follows immediately.

Lemma 2.4. If $i$ is an index for which $c_{i} \geq 0$, then $\liminf |y| \rightarrow \infty|y|^{N-2} u_{i}(y)>0$.

Proof. If $c_{i}<0$ for all $i \in J$ there is nothing to prove. Otherwise, fix an index $i$ for which $c_{i} \geq 0$ and let

$$
\mathcal{O}_{i}=\left\{y \in \mathbb{R}_{+}^{N}: u_{i}(y)<|y|^{2-N}\right\} .
$$

Clearly, to prove Lemma 2.4 it suffices to show $\liminf |y| \rightarrow \infty ; y \in \overline{\mathcal{O}}_{i}|y|^{N-2} u_{i}(y)>0$. For $y \in \overline{\mathcal{O}_{i}}$ we have $u_{i}(y)^{N /(N-2)} \leq|y|^{-2} u_{i}(y)$, so $u_{i}$ satisfies

$$
\begin{cases}-\Delta u_{i}>0 & \text { in } \mathcal{O}_{i} \\ \frac{\partial u_{i}}{\partial y_{N}}-C_{1}|y|^{-2} u_{i}<0 & \text { on } \partial \mathbb{R}_{+}^{N} \cap \overline{\mathcal{O}}_{i}\end{cases}
$$

for some constant $C_{1}=C_{1}\left(\max _{j}\left|c_{j}\right|\right)>0$. For $A \gg 1$ fixed and to be determined, define

$$
\xi(y)=\left|y-A e_{N}\right|^{2-N}+|y|^{1-N} \quad \text { for }|y| \geq 2 A .
$$

By direct computation, one may verify that $\xi$ satisfies

$$
\begin{cases}\Delta \xi>0 & \text { in } \mathbb{R}_{+}^{N} \backslash \bar{B}_{2 A} \\ |y|^{-2} \xi(y) \leq C|y|^{-N} & \text { in } \overline{\mathbb{R}_{+}^{N} \backslash B_{2 A}} \\ \frac{\partial \xi}{\partial y_{N}}(y)=A(N-2)\left|y-A e_{N}\right|^{-N} & \text { on } \partial \mathbb{R}_{+}^{N} \backslash B_{2 A} .\end{cases}
$$

Therefore, we may choose $A=A\left(N, \max _{j}\left|c_{j}\right|\right)$ sufficiently large so that

$$
\left(\frac{\partial}{\partial y_{N}}-C_{1}|y|^{-2}\right) \xi(y)>0 \quad \text { on } \partial \mathbb{R}_{+}^{N} \backslash B_{2 A} \text {. }
$$


Fixing such an $A$ and choosing $\epsilon>0$ small enough to achieve $u_{i}(y)>\epsilon \xi(y)$ on $\left(\partial B_{2 A} \cap \overline{\mathbb{R}_{+}^{N}}\right) \cup\left(\partial \mathcal{O}_{i} \cap\right.$ $\mathbb{R}_{+}^{N}$ ), we obtain

$$
\begin{cases}-\Delta\left(u_{i}-\epsilon \xi\right)>0 & \text { in } \mathcal{O}_{i} \backslash \bar{B}_{2 A} \\ \left(\frac{\partial}{\partial y_{N}}-C_{1}|y|^{-2}\right)\left(u_{i}-\epsilon \xi\right)<0 & \text { on }\left(\partial \mathbb{R}_{+}^{N} \cap \overline{\mathcal{O}}_{i}\right) \backslash B_{2 A} \\ \left(u_{i}-\epsilon \xi\right)(y) \geq 0 & \text { on }\left(\partial B_{2 A} \cap \overline{\mathbb{R}}_{+}^{N}\right) \cup\left(\partial \mathcal{O}_{i} \cap \mathbb{R}_{+}^{N}\right)\end{cases}
$$

Moreover, $\liminf |y| \rightarrow \infty\left(u_{i}-\epsilon \xi\right) \geq 0$, so if $u_{i}-\epsilon \xi$ is negative at some point of $\overline{\mathcal{O}}_{i} \backslash B_{2 A}$, then $u_{i}-\epsilon \xi$ must achieve a negative minimum at some point $\tilde{y} \in \overline{\mathcal{O}}_{i} \backslash B_{2 A}$. By the maximum principle, we may assume $\tilde{y} \in \partial\left(\mathcal{O}_{i} \backslash B_{2 A}\right)$. The third item of (2.7) imposes $\tilde{y} \in\left(\partial \mathbb{R}_{+}^{N} \cap \overline{\mathcal{O}}_{i}\right) \backslash B_{2 A}$. On the other hand, $\left(u_{i}-\epsilon \xi\right)(\tilde{y})<0$ and $\frac{\partial}{\partial y_{N}}\left(u_{i}-\epsilon \xi\right)(\tilde{y}) \geq 0$, so the second item of (2.7) is violated. We conclude that $u_{i}-\epsilon \xi \geq 0$ in $\overline{\mathcal{O}}_{i} \backslash B_{2 A}$. Consequently,

$$
\liminf _{|y| \rightarrow \infty ; y \in \overline{\mathcal{O}}_{i}}|y|^{N-2} u_{i}(y) \geq \epsilon \liminf _{|y| \rightarrow \infty}|y|^{N-2} \xi(y)>0 .
$$

Lemma 2.4 is established.

Proof of Proposition 2.1. Let $r_{0}$ be as in Lemma 2.2. By Lemmas 2.3 and 2.4 we may first choose $c_{0} \in(0,1]$ such that

$$
u_{i}(y) \geq c_{0}|y|^{2-N} \quad \text { for all } y \in \overline{\mathbb{R}_{+}^{N}} \backslash B_{r_{0}} \quad \text { and all } i \in J
$$

and then choose $\lambda_{0} \in\left(0, r_{0}\right)$ such that

$$
\lambda_{0}^{N-2}\left(\max _{j} \frac{\max }{B_{r_{0}}^{+}} u_{j}\right) \leq c_{0}
$$

For such $\lambda_{0}$, if $0<\lambda \leq \lambda_{0}$ then

$$
u_{i, \lambda}(y) \leq \lambda_{0}^{N-2}\left(\max _{j} \frac{\max }{B_{r_{0}}^{+}} u_{j}\right)|y|^{2-N} \leq c_{0}|y|^{2-N} \leq u_{i}(y) \quad \text { for all } y \in \overline{\mathbb{R}_{+}^{N}} \backslash B_{r_{0}} \text { and all } i \in J .
$$

Combining this with Lemma 2.2 establishes Proposition 2.1.

\section{A Symmetry Relation for $u_{1}, \cdots, u_{m}$}

In this section we prove the following proposition.

Proposition 3.1. For each $x \in \partial \mathbb{R}_{+}^{N}, \bar{\lambda}(x)<\infty$ and

$$
w_{i, x, \bar{\lambda}(x)}(y) \equiv 0 \quad \text { for all } y \in \overline{\mathbb{R}_{+}^{N}} \backslash\{x\} \text { and all } i \in J .
$$

For convenience Proposition 3.1 will be proven for $x=0$ only. Proposition 3.1 will be established with the aid of some lemmas.

Lemma 3.2. Let $\mathcal{A}$ be a matrix satisfying (1.3) and let $x_{0} \in \partial \mathbb{R}_{+}^{N}$. For $\lambda \in\left(0, \bar{\lambda}\left(x_{0}\right)\right]$, if there exists $i_{0} \in J$ for which $w_{i_{0}, x_{0}, \lambda} \equiv 0$ in $\Sigma_{x_{0}, \lambda}$, then

$$
w_{i, x_{0}, \lambda} \equiv 0 \quad \text { in } \overline{\mathbb{R}_{+}^{N}} \backslash\left\{x_{0}\right\} \text { for all } i \in J .
$$


Proof. Clearly, it suffices to show that the equality in (3.1) holds for all $y \in \Sigma_{x_{0}, \lambda}$. The proof is given for $x=0$ only. The proof for general $x_{0} \in \partial \mathbb{R}_{+}^{N}$ is similar. Fix $0<\lambda \leq \bar{\lambda}$. According to (2.2), the interior equation for $w_{i, \lambda}$ may be written

$$
-\Delta w_{i, \lambda}=\sum_{j=1}^{m} \phi_{i j}\left(u_{j}^{a_{i j}}-u_{j, \lambda}^{a_{i j}}\right) \quad \text { in } \Sigma_{\lambda} \text { for all } i \in J,
$$

where

$$
\phi_{i j}=\left(\prod_{k=1}^{j-1} u_{k, \lambda}^{a_{i k}}\right)\left(\prod_{\ell=j+1}^{m} u_{\ell}^{a_{i \ell}}\right)>0 .
$$

Here the notational conventions $\prod_{k=1}^{0} u_{k, \lambda}^{a_{i k}}=1$ and $\prod_{\ell=m+1}^{m} u_{\ell}^{a_{i \ell}}=1$ are used. Lemma 3.2 now follows from the irreducibility of $\mathcal{A}$ and since $w_{j, \lambda} \geq 0$ in $\Sigma_{\lambda}$ for all $j \in J$.

Lemma 3.3. If $x_{0} \in \partial \mathbb{R}_{+}^{N}$ with $\bar{\lambda}\left(x_{0}\right)<\infty$, then $w_{i, x_{0}, \bar{\lambda}\left(x_{0}\right)} \equiv 0$ in $\overline{\mathbb{R}_{+}^{N}} \backslash\left\{x_{0}\right\}$ for all $i \in J$.

Proof. For simplicity, we assume $x_{0}=0$. By Lemma 3.2, it suffices to show that there exists $i \in J$ such that $w_{i, \bar{\lambda}} \equiv 0$ in $\overline{\mathbb{R}_{+}^{N}} \backslash\{0\}$. In fact, we only need to show this equality holds in $\Sigma_{\bar{\lambda}}$ for some $i \in J$. For the sake of obtaining a contradiction, suppose that for all $i \in J$, there is some point of $\Sigma_{\bar{\lambda}}$ at which $w_{i, \bar{\lambda}}$ is positive. By the maximum principle we have

$$
w_{i, \bar{\lambda}}(y)>0 \quad \text { for all } y \in \Sigma_{\bar{\lambda}} \text { and all } i \in J
$$

Moreover,

$$
w_{i, \bar{\lambda}}(y)>0 \quad \text { for all } y \in \partial \Sigma_{\bar{\lambda}} \backslash \partial B_{\bar{\lambda}} \text { and all } i \in J .
$$

Indeed, if $\tilde{y} \in \partial \Sigma_{\bar{\lambda}} \backslash \partial B_{\bar{\lambda}}$ and $i_{0} \in J$ are such that with $w_{i_{0}, \bar{\lambda}}(\tilde{y})=0$, then apply Hopf's Lemma to $w_{i_{0}, \bar{\lambda}}$ on any ball $B \subset \Sigma_{\bar{\lambda}}$ such that $\partial B \cap \partial \Sigma_{\bar{\lambda}}=\{\tilde{y}\}$ to deduce

$$
\frac{\partial w_{i_{0}, \bar{\lambda}}}{\partial y_{N}}(\tilde{y})>0
$$

On the other hand, if $c_{i_{0}}<0$ then

$$
\frac{\partial w_{i_{0}, \bar{\lambda}}}{\partial y_{N}}(\tilde{y})=c_{i_{0}}\left(\prod_{j=1}^{m} u_{j}(\tilde{y})^{b_{i_{0} j}}-\prod_{j=1}^{m} u_{j, \bar{\lambda}}(\tilde{y})^{b_{i_{0} j}}\right) \leq 0 .
$$

If $c_{i_{0}} \geq 0$, then

$$
\frac{\partial w_{i_{0}, \bar{\lambda}}}{\partial y_{N}}(\tilde{y})=c_{i_{0}}\left(u_{i_{0}}(\tilde{y})^{N /(N-2)}-u_{i_{0}, \bar{\lambda}}(\tilde{y})^{N /(N-2)}\right)=0 .
$$

In either case, (3.5) is violated, so (3.4) holds. $\left.\bar{\Sigma}_{\bar{\lambda}}\right)$.

Now, for $y \in \partial B_{\bar{\lambda}} \cap \partial \Sigma_{\bar{\lambda}}$, let $\nu=\nu(y)$ denote the unit outer normal vector to $B_{\bar{\lambda}}$ (pointing into

Claim 3.4. There exists $\epsilon>0$ such that

$$
\frac{\partial w_{i, \bar{\lambda}}}{\partial \nu}(y) \geq \epsilon \quad \text { for all } y \in \partial \Sigma_{\bar{\lambda}} \cap \partial B_{\bar{\lambda}} \text { and all } i \in J \text {. }
$$


Proof. In view of (3.3) and (2.3), a routine application of Hopf's Lemma yields the positivity of $\frac{\partial w_{i, \bar{\lambda}}}{\partial \nu}(y)$ for all $y \in \partial \Sigma_{\bar{\lambda}} \backslash \partial \mathbb{R}_{+}^{N}$ and all $i \in J$. Since $\partial \Sigma_{\bar{\lambda}} \cap \partial B_{\bar{\lambda}}$ is compact, Claim 3.4 will be established once we show

$$
\frac{\partial w_{i, \bar{\lambda}}}{\partial \nu}(y)>0 \quad \text { for all } y \in \partial B_{\bar{\lambda}} \cap \partial \mathbb{R}_{+}^{N} \text { and all } i \in J
$$

To show this, define

and

$$
\Omega=\left\{y \in \Sigma_{\bar{\lambda}}: \operatorname{dist}\left(y, \partial B_{\bar{\lambda}} \cap \partial \mathbb{R}_{+}^{N}\right)<\frac{\bar{\lambda}}{2}\right\}
$$

$$
\phi(y)=\delta e^{\alpha y_{N}}\left(|y|^{2}-\bar{\lambda}^{2}\right),
$$

where $\delta>0$ (small) and $\alpha>0$ (large) are positive constants which are to be determined. Elementary computations yield

$$
\begin{cases}\Delta \phi>0 & \text { in } \mathbb{R}_{+}^{N} \\ \phi \equiv 0 & \text { on } \partial B_{\bar{\lambda}} \\ \frac{\partial \phi}{\partial y_{N}}=\alpha \phi & \text { on } \partial \mathbb{R}_{+}^{N} \\ \frac{\partial \phi}{\partial \nu}=2 \delta \bar{\lambda} e^{\alpha y_{N}} & \text { on } \partial B_{\bar{\lambda}} .\end{cases}
$$

Moreover, if $i$ is an index for which $c_{i}<0$, then by using each of the second item of (2.2), (3.4) and the third item of (3.7) one may verify that for any choice of $\alpha>0$

$$
\frac{\partial}{\partial y_{N}}\left(w_{i, \bar{\lambda}}-\phi\right) \leq-\alpha \phi \leq \frac{\alpha}{2}\left(w_{i, \lambda}-\phi\right) \quad \text { on } \partial \Omega \cap \partial \mathbb{R}_{+}^{N} .
$$

If $i$ is an index for which $c_{i} \geq 0$, then by the Mean-Value Theorem, there is $\psi_{i}(y) \in\left[u_{i, \bar{\lambda}}(y), u_{i}(y)\right]$ such that

$$
\begin{aligned}
\frac{\partial}{\partial y_{N}}\left(w_{i, \bar{\lambda}}-\phi\right) & =c_{i}\left(u_{i}^{N /(N-2)}-u_{i, \bar{\lambda}}^{N /(N-2)}\right)-\alpha \phi \\
& =\frac{N}{N-2} c_{i} \psi_{i}^{2 /(N-2)} w_{i, \bar{\lambda}}-\alpha \phi \\
& \leq \frac{N}{N-2}\left(\max _{j}\left|c_{j}\right|\right)\left(\max _{j} \max _{\bar{\Omega}} u_{j}\right)^{2 /(N-2)} w_{i, \bar{\lambda}}-\alpha \phi .
\end{aligned}
$$

Therefore, by choosing $\alpha=\alpha\left(N, \max _{j}\left|c_{j}\right|, \max _{j} \max _{\bar{\Omega}} u_{j}\right)$ sufficiently large, we obtain

$$
\frac{\partial}{\partial y_{N}}\left(w_{i, \bar{\lambda}}-\phi\right) \leq \frac{\alpha}{2}\left(w_{i, \bar{\lambda}}-\phi\right) \quad \text { on } \partial \Omega \cap \partial \mathbb{R}_{+}^{N} .
$$

Combining (3.8) and (3.9) we see that there is a constant $C_{1}>0$ for which

$$
\frac{\partial}{\partial y_{N}}\left(w_{i, \bar{\lambda}}-\phi\right) \leq C_{1}\left(w_{i, \bar{\lambda}}-\phi\right) \quad \text { on } \partial \Omega \cap \partial \mathbb{R}_{+}^{N} \text { for all } i \in J .
$$

Fix any such $C_{1}$. After choosing $\delta$ sufficiently small $w_{i, \bar{\lambda}}-\phi$ is seen to satisfy

$$
\left\{\begin{array}{ll}
-\Delta\left(w_{i, \bar{\lambda}}-\phi\right)>0 & \text { in } \Omega \\
w_{i, \bar{\lambda}}-\phi \equiv 0 & \text { on } \partial \Omega \cap \partial B_{\bar{\lambda}} \\
w_{i, \bar{\lambda}}-\phi>0 & \text { on } \partial \Omega \backslash \partial \Sigma_{\bar{\lambda}}
\end{array} \quad \text { for all } i \in J .\right.
$$


By the maximum principle, if there exists $i_{0} \in J$ such that $w_{i_{0}, \bar{\lambda}}-\phi$ is negative at some point of $\bar{\Omega}$ then $w_{i_{0}, \bar{\lambda}}-\phi$ achieves a negative minimum value over $\bar{\Omega}$ at some point $\tilde{y} \in \partial \Omega$. By the second and third items of (3.11), we may assume $\tilde{y} \in \partial \mathbb{R}_{+}^{N} \cap\{y: \bar{\lambda}<|y| \leq 3 \bar{\lambda} / 2\}$. Since $\tilde{y}$ is a minimizer of $w_{i_{0}, \bar{\lambda}}-\phi$ and by (3.10), we have

$$
0 \leq \frac{\partial}{\partial y_{N}}\left(w_{i_{0}, \bar{\lambda}}-\phi\right)(\tilde{y}) \leq C_{1}\left(w_{i_{0}, \bar{\lambda}}-\phi\right)(\tilde{y})<0,
$$

a contradiction. We conclude that $w_{i, \bar{\lambda}} \geq \phi$ in $\bar{\Omega}$ for all $i \in J$. In particular, $\frac{\partial w_{i, \bar{\lambda}}}{\partial \nu} \geq \frac{\partial \phi}{\partial \nu}$ on $\partial B_{\bar{\lambda}} \cap \partial \mathbb{R}_{+}^{N}$ for all $i \in J$. Combining this with the last item of (3.7), we obtain inequality (3.6). Claim 3.4 follows.

In view of Claim 3.4 and the continuity of $\lambda \mapsto w_{i, \lambda}$, we may choose $R_{0}>\bar{\lambda}$ such that

$$
\frac{\partial w_{i, \lambda}}{\partial r}(y) \geq \frac{\epsilon}{2} \quad \text { for all } y \in \overline{B_{R_{0}}^{+}} \backslash B_{\lambda}, \text { all } \lambda \in\left[\bar{\lambda}, R_{0}\right] \text { and all } i \in J \text {. }
$$

Therefore,

$$
w_{i, \lambda}(y)>0 \quad \text { in } \overline{B_{R_{0}}^{+}} \backslash \bar{B}_{\lambda} \text { for all } \lambda \in\left[\bar{\lambda}, R_{0}\right] \text { and all } i \in J .
$$

Claim 3.5. If $i$ is an index for which $c_{i}<0$, then $\liminf _{|y| \rightarrow \infty}|y|^{N-2} w_{i, \bar{\lambda}}(y)>0$.

Proof. If $c_{i} \geq 0$ for all $i \in J$, there is nothing to prove. Otherwise, let $i$ be an index for which $c_{i}<0$ and define

$$
h_{i}(y)=\left(\min _{\partial B_{R_{0}} \cap \overline{\mathbb{R}_{+}^{N}}} w_{i, \bar{\lambda}}\right) R_{0}^{N-2}|y|^{2-N} \quad \text { for }|y| \geq R_{0} .
$$

By performing elementary computations using (3.3), (3.4) and the negativity of $c_{i}$, one may verify that $w_{i, \bar{\lambda}}-h_{i}$ satisfies

$$
\begin{cases}-\Delta\left(w_{i, \bar{\lambda}}-h_{i}\right) \geq 0 & \text { in } \mathbb{R}_{+}^{N} \backslash \bar{B}_{R_{0}} \\ w_{i, \bar{\lambda}}-h_{i} \geq 0 & \text { on } \partial B_{R_{0}} \cap \overline{\mathbb{R}_{+}^{N}} \\ \frac{\partial}{\partial y_{N}}\left(w_{i, \bar{\lambda}}-h_{i}\right)=c_{i}\left(\prod_{j=1}^{m} u_{j}^{a_{i j}}-\prod_{j=1}^{m} u_{j, \bar{\lambda}}^{a_{i j}}\right)<0 & \text { on } \partial \mathbb{R}_{+}^{N} \backslash B_{R_{0}} .\end{cases}
$$

Moreover, using (3.3) once again we have

$$
\liminf _{|y| \rightarrow \infty}\left(w_{i, \bar{\lambda}}-h_{i}\right)(y) \geq 0 .
$$

Consequently, if $w_{i, \bar{\lambda}}-h_{i}$ is negative at some point of $\overline{\mathbb{R}_{+}^{N}} \backslash B_{R_{0}}$, then $w_{i, \bar{\lambda}}-h_{i}$ attains a negative minimum value over $\overline{\mathbb{R}_{+}^{N}} \backslash B_{R_{0}}$ at some point $\tilde{y} \in \overline{\mathbb{R}_{+}^{N}} \backslash B_{R_{0}}$. By the maximum principle, we may assume $\tilde{y} \in \partial\left(\mathbb{R}_{+}^{N} \backslash B_{R_{0}}\right)$. By the second item of (3.13) we must have $\tilde{y} \in \partial \mathbb{R}_{+}^{N} \backslash \bar{B}_{R_{0}}$. On the other hand, since $\tilde{y}$ minimizes $w_{i, \bar{\lambda}}-h_{i}$ and by the third item of (3.13) we have

$$
0 \leq \frac{\partial}{\partial y_{N}}\left(w_{i, \bar{\lambda}}-h_{i}\right)(\tilde{y})<0,
$$

a contradiction. We conclude that $w_{i, \bar{\lambda}} \geq h_{i}$ in $\mathbb{R}_{+}^{N} \backslash B_{R_{0}}$. Claim 3.5 follows immediately.

Claim 3.6. If $i$ is an index for which $c_{i} \geq 0$, then $\liminf _{|y| \rightarrow \infty}|y|^{N-2} w_{i, \bar{\lambda}}(y)>0$. 
Proof. The proof is similar to the proof of Lemma 2.4. Suppose $i$ is an index for which $c_{i} \geq 0$ and set

$$
\mathcal{O}_{i}=\left\{y \in \Sigma_{\bar{\lambda}}: w_{i, \bar{\lambda}}(y)<u_{i, \bar{\lambda}}(y)\right\}
$$

To prove Claim 3.6, it suffices to show that

$$
\liminf _{|y| \rightarrow \infty ; y \in \overline{\mathcal{O}}_{i}}|y|^{N-2} w_{i, \bar{\lambda}}(y)>0
$$

We have

$$
u_{i}(y) \leq 2 \bar{\lambda}^{N-2}\left(\max _{j} \frac{\max }{B_{\bar{\lambda}}^{ \pm}} u_{j}\right)|y|^{2-N} \quad \text { for all } y \in \mathcal{O}_{i} .
$$

According to the Mean-Value Theorem, there is $\psi_{i}(y) \in\left[u_{i, \bar{\lambda}}(y), u_{i}(y)\right]$ such that for all $y \in \partial \Sigma_{\bar{\lambda}} \cap$ $\partial \mathbb{R}_{+}^{N}$,

$$
\begin{aligned}
u_{i}(y)^{N /(N-2)}-u_{i, \bar{\lambda}}(y)^{N /(N-2)} & =\frac{N}{N-2} \psi_{i}(y)^{2 /(N-2)} w_{i, \bar{\lambda}}(y) \\
& \leq \frac{N}{N-2} u_{i}(y)^{2 /(N-2)} w_{i, \bar{\lambda}}(y) .
\end{aligned}
$$

Therefore, using the boundary equation for $w_{i, \bar{\lambda}}$ in (2.2) corresponding to $c_{i} \geq 0$ and using inequality (3.15), there is a constant $C_{1}=C_{1}\left(N, \bar{\lambda}, \max _{j}\left|c_{j}\right|, \max _{j} \max _{\bar{B}_{\bar{\lambda}}^{+}} u_{j}\right)>0$ such that

$$
\left(\frac{\partial}{\partial y_{N}}-C_{1}|y|^{-2}\right) w_{i, \bar{\lambda}} \leq 0 \quad \text { for all } y \in \overline{\mathcal{O}}_{i} \cap \partial \mathbb{R}_{+}^{N} .
$$

For $A \gg 1$ large and to be determined, let $\xi(y)$ be as in (2.5). Then $\xi$ still satisfies (2.6) and by choosing $A$ sufficiently large (and depending on $C_{1}$ ) we may achieve

$$
\left(\frac{\partial}{\partial y_{N}}-C_{1}|y|^{-2}\right) \xi(y)>0 \quad \text { on } \partial \mathbb{R}_{+}^{N} \backslash B_{2 A}
$$

Fix any such $A$ and choose $\epsilon>0$ sufficiently small so that

$$
\left(w_{i, \bar{\lambda}}-\epsilon \xi\right)(y)>0 \quad \text { on }\left(\partial B_{2 A} \cap \overline{\mathbb{R}_{+}^{N}}\right) \cup\left(\partial \mathcal{O}_{i} \cap \mathbb{R}_{+}^{N}\right) .
$$

Then

$$
\begin{cases}-\Delta\left(w_{i, \bar{\lambda}}-\epsilon \xi\right)>0 & \text { in } \mathcal{O}_{i} \backslash \bar{B}_{2 A} \\ \left(w_{i, \bar{\lambda}}-\epsilon \xi\right)>0 & \text { on } \partial\left(\mathcal{O}_{i} \backslash B_{2 A}\right) \backslash \partial \mathbb{R}_{+}^{N} \\ \left(\frac{\partial}{\partial y_{N}}-C_{1}|y|^{-2}\right)\left(w_{i, \bar{\lambda}}-\epsilon \xi\right)<0 & \text { on }\left(\overline{\mathcal{O}}_{i} \backslash B_{2 A}\right) \cap \partial \mathbb{R}_{+}^{N}\end{cases}
$$

Moreover, $\liminf f_{|y| \rightarrow \infty}\left(w_{i, \bar{\lambda}}-\epsilon \xi\right)(y) \geq 0$. Claim 3.6 now follows by the argument in the proof of Lemma 2.4 .

In view of Claims 3.5 and 3.6 and with $R_{0}$ as in (3.12) we may choose $c_{0}>0$ such that

$$
w_{i, \bar{\lambda}}(y) \geq c_{0}|y|^{2-N} \quad \text { for all } y \in \overline{\mathbb{R}_{+}^{N}} \backslash B_{R_{0}} \text { and all } i \in J .
$$

Therefore, for any $\lambda>0$ and any $i \in J$ we have 


$$
w_{i, \lambda}(y) \geq c_{0}|y|^{2-N}+\left(\bar{\lambda}^{N-2} u_{i}\left(\frac{\bar{\lambda}^{2} y}{|y|^{2}}\right)-\lambda^{N-2} u_{i}\left(\frac{\lambda^{2} y}{|y|^{2}}\right)\right)|y|^{2-N} \quad \text { for all } y \in \overline{\mathbb{R}_{+}^{N}} \backslash B_{R_{0}} .
$$

By uniform continuity of $u_{i}$ on $\bar{B}_{R_{0}}^{+}$, there exists $\epsilon_{0} \in\left(0, R_{0}-\bar{\lambda}\right)$ such that

$$
\left|\bar{\lambda}^{N-2} u_{i}\left(\frac{\bar{\lambda}^{2} y}{|y|^{2}}\right)-\lambda^{N-2} u_{i}\left(\frac{\lambda^{2} y}{|y|^{2}}\right)\right|<\frac{c_{0}}{2} \quad \text { for all } y \in \overline{\mathbb{R}_{+}^{N}} \backslash B_{R_{0}} \text {, all } \lambda \in\left[\bar{\lambda}, \bar{\lambda}+\epsilon_{0}\right] \text { and all } i \in J \text {. }
$$

Using this estimate in inequality (3.17), we conclude that

$$
w_{i, \lambda}(y)>\frac{c_{0}}{2}|y|^{2-N} \quad \text { for all } y \in \overline{\mathbb{R}_{+}^{N}} \backslash B_{R_{0}} \text {, all } \lambda \in\left[\bar{\lambda}, \bar{\lambda}+\epsilon_{0}\right] \text { and all } i \in J .
$$

Combining this estimate with (3.12), we conclude that $w_{i, \lambda}(y) \geq 0$ in $\overline{\mathbb{R}_{+}^{N}} \backslash B_{\lambda}$ for all $\lambda \in\left[\bar{\lambda}, \bar{\lambda}+\epsilon_{0}\right]$ and all $i \in J$. This contradicts the definition of $\bar{\lambda}$. Lemma 3.3 is established.

Lemma 3.7. If there exists $x_{0} \in \partial \mathbb{R}_{+}^{N}$ for which $\bar{\lambda}\left(x_{0}\right)=\infty$, then $\bar{\lambda}(x)=\infty$ for all $x \in \partial \mathbb{R}_{+}^{N}$.

Proof. Suppose $x_{0} \in \partial \mathbb{R}_{+}^{N}$ is such that $\bar{\lambda}\left(x_{0}\right)=\infty$. By definition of $\bar{\lambda}\left(x_{0}\right)$, for all $\lambda>0$ we have

$$
u_{i}(y) \geq\left(\frac{\lambda}{\left|y-x_{0}\right|}\right)^{N-2} u_{i}\left(x_{0}+\frac{\lambda^{2}\left(y-x_{0}\right)}{\left|y-x_{0}\right|^{2}}\right) \quad \text { in } \Sigma_{x_{0}, \lambda} \text { for all } i \in J .
$$

Consequently, $|y|^{N-2} u_{i}(y) \rightarrow \infty$ as $|y| \rightarrow \infty$ for all $i \in J$. Now suppose $x \in \partial \mathbb{R}_{+}^{N}$ is such that $\bar{\lambda}(x)<\infty$. By Lemma 3.3, $u_{i}=u_{i, x, \bar{\lambda}(x)}$ on $\overline{\mathbb{R}_{+}^{N}} \backslash\{x\}$ for all $i \in J$. Multiplying this equality by $|y|^{N-2}$ and letting $|y| \rightarrow \infty$ we obtain

$$
|y|^{N-2} u_{i}(y) \rightarrow \bar{\lambda}(x)^{N-2} u_{i}(x)<\infty \quad \text { for all } i \in J
$$

which is a contradiction.

Lemma 3.8. For each $x \in \partial \mathbb{R}_{+}^{N}, \bar{\lambda}(x)<\infty$.

Proof. If Lemma 3.8 fails, then by Lemma 3.7, we have $\bar{\lambda}(x)=\infty$ for all $x \in \partial \mathbb{R}_{+}^{N}$. By Lemma [5.2, we see that for all $i \in J, u_{i}(y)$ depends only on $y_{N}$. In this case, (1.9) becomes

$$
\left\{\begin{array}{ll}
u_{i}^{\prime \prime}(t)=-\prod_{j=1}^{m} u_{j}(t)^{a_{i j}} & \text { in }(0, \infty) \\
u_{i}^{\prime}(0)=c_{i} \prod_{j=1}^{m} u_{j}(0)^{b_{i j}} & \\
u_{i}(t)>0 & \text { on }[0, \infty)
\end{array} \quad \text { for all } i \in J .\right.
$$

Combining the first and third items of (3.18), we see that $u_{i}^{\prime}$ is strictly decreasing in $(0, \infty)$ for all $i \in J$.

Now, observe that there is no index $i_{0} \in J$ for which $u_{i_{0}}^{\prime}(0)=0$. Indeed, if such an $i_{0}$ were to exist then since $u_{i_{0}}^{\prime}$ is strictly decreasing, we would have $u_{i_{0}}^{\prime}(1)<0$. By choosing $t$ sufficiently large we could achieve

$$
u_{i_{0}}(t)=u_{i_{0}}(1)+\int_{1}^{t} u_{i_{0}}^{\prime}(s) d s \leq u_{i_{0}}(1)+u_{i_{0}}^{\prime}(1)(t-1)<0,
$$

which contradicts the third item of (3.18). By a similar argument, we see that there is no index $i_{0} \in J$ for which $u_{i_{0}}^{\prime}(0)<0$. Therefore, we must have $u_{i}^{\prime}(0)>0$ for all $i \in J$. Moreover, by an argument similar to the above, we see that 


$$
u_{i}^{\prime}(t)>0 \quad \text { for all } t \in[0, \infty) \text { and all } i \in J .
$$

In particular, $u_{i}^{\prime}$ is decreasing and bounded below by zero, so

$$
\ell_{i}=\lim _{t \rightarrow \infty} u_{i}^{\prime}(t)
$$

exists and is non-negative for all $i \in J$. Since both $u_{i}(0)>0$ and $u_{i}^{\prime}(t)>0$ in $[0, \infty)$ for all $i \in J$, there exists $\epsilon>0$ such that

$$
u_{i}(t) \geq \epsilon \quad \text { for all } t \in[0, \infty) \text { and all } i \in J .
$$

On the other hand, by the first equality of (3.18), we have

$$
u_{i}^{\prime}(t)-u_{i}^{\prime}(0)=-\int_{0}^{t} \prod_{j=1}^{m} u_{j}(s)^{a_{i j}} d s .
$$

Letting $t \rightarrow \infty$ in this equation we obtain

$$
u_{i}^{\prime}(0)-\ell_{i}=\int_{0}^{\infty} \prod_{j=1}^{m} u_{j}(s)^{a_{i j}} d s
$$

so that $\prod_{j=1}^{m} u_{j}^{a_{i j}} \in L^{1}(0, \infty)$. In particular, this integrability provides the existence of $i_{0} \in J$ for which $\liminf t \rightarrow \infty u_{i_{0}}(t)=0$. This contradicts (3.19). Lemma 3.8 is established.

Proof of Proposition [3.1. Combine the results of Lemmas 3.3 and 3.8.

\section{Completion of the Proof of Theorem 1.3}

By Proposition 3.1, for all $x \in \partial \mathbb{R}_{+}^{N}$, we have both $\bar{\lambda}(x)<\infty$ and

$$
u_{i}(y)=\left(\frac{\bar{\lambda}(x)}{|y-x|}\right)^{N-2} u_{i}\left(x+\frac{\bar{\lambda}(x)^{2}(y-x)}{|y-x|^{2}}\right) \quad \text { in } \overline{\mathbb{R}_{+}^{N}} \backslash\{x\} \text { for all } i \in J .
$$

Restricting this equality to $\mathbb{R}^{N-1}=\partial \mathbb{R}_{+}^{N}$, writing $y=y^{\prime}+y_{N} e_{N}$ with $y^{\prime} \in \partial \mathbb{R}_{+}^{N}$ and applying Lemma 5.3 on $\mathbb{R}^{N-1}$, for each $i \in J$ we obtain $A_{i} \geq 0, d_{i}>0$ and $\bar{x}_{i} \in \partial \mathbb{R}_{+}^{N}$ such that

$$
u_{i}\left(y^{\prime}\right)=\frac{A_{i}}{\left(d_{i}^{2}+\left|y^{\prime}-\bar{x}_{i}\right|^{2}\right)^{(N-2) / 2}} \quad \text { for all } y^{\prime} \in \partial \mathbb{R}_{+}^{N} .
$$

By this expression and by (4.1), it is easy to see that

$$
A_{i}=\lim _{\left|y^{\prime}\right| \rightarrow \infty}\left|y^{\prime}\right|^{N-2} u_{i}\left(y^{\prime}\right)=\bar{\lambda}(x)^{N-2} u_{i}(x)>0 \quad \text { for all } x \in \partial \mathbb{R}_{+}^{N} .
$$

Next, observe that

$$
d_{i}=d_{j} \quad \text { and } \quad \bar{x}_{i}=\bar{x}_{j} \quad \text { for all }(i, j) \in J \times J .
$$

Indeed, by (4.3) we have

$$
\frac{u_{i}(x)}{A_{i}}=\frac{u_{j}(x)}{A_{j}} \quad \text { for all } x \in \partial \mathbb{R}_{+}^{N} \text { and all }(i, j) \in J \times J .
$$

In view of (4.2), the above equality yields

$$
d_{i}^{2}+\left|x-\bar{x}_{i}\right|^{2}=d_{j}^{2}+\left|x-\bar{x}_{j}\right|^{2} \quad \text { for all } x \in \partial \mathbb{R}_{+}^{N} \text { and all }(i, j) \in J \times J .
$$


The equalities in (4.4) follow immediately.

Returning to (4.2) with (4.4), and using $d$ to denote the common value of $d_{i}$ and $\bar{x}$ to denote the common value of $\bar{x}_{i}$, we obtain

$$
u_{i}(x)=\frac{A_{i}}{\left(d^{2}+|x-\bar{x}|^{2}\right)^{(N-2) / 2}} \quad \text { for all } x \in \partial \mathbb{R}_{+}^{N} \text { and all } i \in J .
$$

Now that we know the form of the restriction of $u_{i}$ to $\partial \mathbb{R}_{+}^{N}$ known, we wish to deduce the form of $u_{i}$. To achieve this we follow the arguments of [4], [2], [17]. Using (4.3) to replace $A_{i}$ in (4.5), we see that

$$
\bar{\lambda}(x)^{2}=d^{2}+|x-\bar{x}|^{2} \quad \text { for all } x \in \partial \mathbb{R}_{+}^{N} .
$$

Setting $Q=\bar{x}+d e_{N}$ and $P=\bar{x}-d e_{N}$, equation (4.6) says that for each $x \in \partial \mathbb{R}_{+}^{N}, \partial B(x, \bar{\lambda}(x))$ contains both $P$ and $Q$.

Next, for $y \in \mathbb{R}^{N}$ consider

$$
T y=P+\frac{4 d^{2}(y-P)}{|y-P|^{2}},
$$

the conformal inversion of $y$ about $\partial B(P, 2 d)$. By performing elementary computations, one may verify that $T$ enjoys the following properties.

(i) $T=T^{-1}$ on $\mathbb{R}^{N} \cup\{\infty\}$

(ii) $T\left(\mathbb{R}_{+}^{N}\right)=B(Q, 2 d)$

(iii) For each $x \in \partial \mathbb{R}_{+}^{N}$, the image of $\partial B(x, \bar{\lambda}(x))$ under $T$ is the hyperplane $\mathcal{H}(x)$ through $Q$ that is orthogonal to $x-P$.

(iv) If $z$ and $\tilde{z}$ are symmetric about $\mathcal{H}(x)$, then $T z$ and $T \tilde{z}$ are symmetric about $\partial B(x, \bar{\lambda}(x))$ in the sense that

$$
T \tilde{z}=x+\frac{\bar{\lambda}(x)^{2}(T z-x)}{|T z-x|^{2}} .
$$

See Figure 4.1 for a visual representation of the mapping properties of $T$.

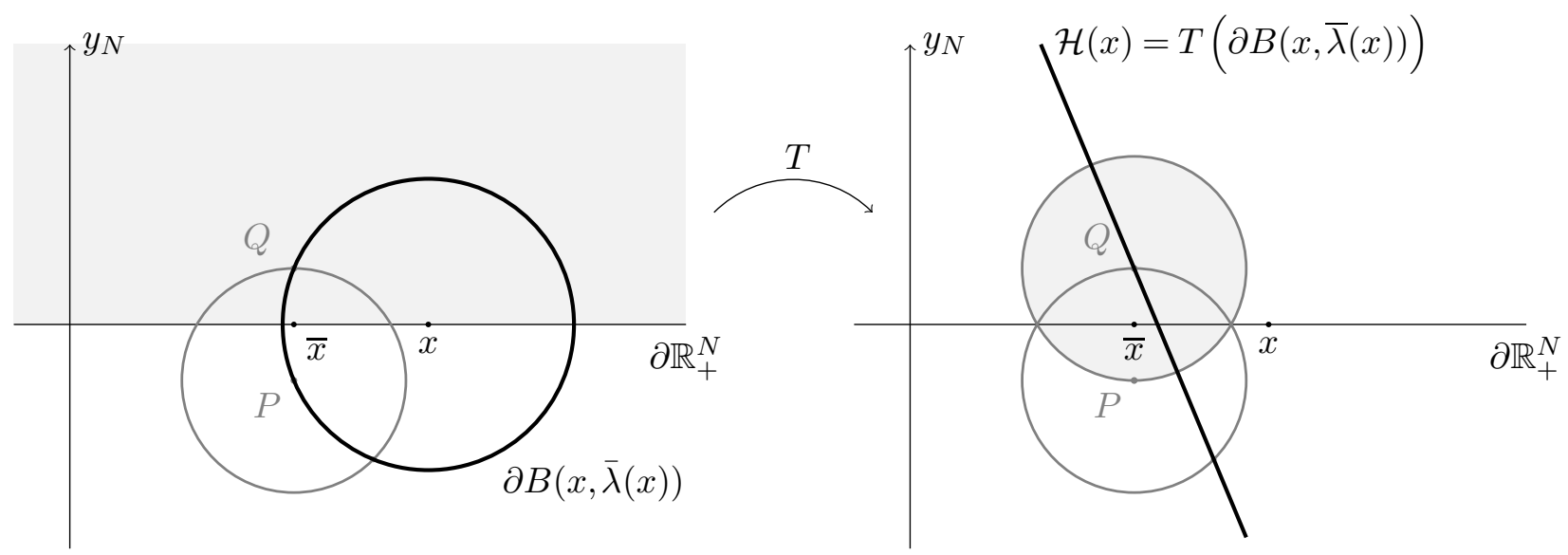

FiguRE 4.1. Visual representation of the properties of $T$ 
For $z \in B(Q, 2 d)$ and $i \in J$, define

$$
v_{i}(z)=\left(\frac{2 d}{|z-P|}\right)^{N-2} u_{i}(T z) .
$$

If $x \in \partial \mathbb{R}_{+}^{N}$, since $u_{i}$ is symmetric about $\partial B(x, \bar{\lambda}(x))$ in the sense of equation (4.1), $v_{i}$ is symmetric about $\mathcal{H}(x)$ in $B(Q, 2 d)$. Indeed, fix $x \in \partial \mathbb{R}_{+}^{N}$ and suppose $z, \tilde{z} \in B(Q, 2 d)$ are symmetric about $\mathcal{H}(x)$. By performing elementary computations using equations (4.1) and (4.7) we obtain

$$
v_{i}(z)=\left(\frac{2 d}{|z-P|}\right)^{N-2}\left(\frac{\bar{\lambda}(x)}{|T z-x|}\right)^{N-2} u_{i}(T \tilde{z})=v_{i}(\tilde{z}) .
$$

Since this holds for all $x \in \partial \mathbb{R}_{+}^{N}, v_{i}$ is radially symmetric about $Q$ in $B(Q, 2 d)$.

Next, observe that the definition of $v_{i}$ may be extended to $P$ such that the resulting extension is continuous. Indeed, writing $y=T z$ for $z \in B(Q, 2 d)$ and using (4.1) with $x=\bar{x}$ we have

$$
\begin{aligned}
v_{i}(z) & =\left(\frac{|y-P|}{2 d}\right)^{N-2} u_{i}(y) \\
& =\left(\frac{|y-P|}{2 d}\right)^{N-2}\left(\frac{\bar{\lambda}(\bar{x})}{|y-\bar{x}|}\right)^{N-2} u_{i}\left(\bar{x}+\frac{\bar{\lambda}(\bar{x})^{2}(y-\bar{x})}{|y-\bar{x}|^{2}}\right) .
\end{aligned}
$$

Letting $z \rightarrow P$ from within $\bar{B}(Q, 2 d) \backslash\{P\}$ (so that $y \rightarrow \infty$ from within $\overline{\mathbb{R}_{+}^{N}}$ ) in this equality and using $\bar{\lambda}(\bar{x})=d$ gives

$$
\lim _{z \rightarrow P ; z \in \bar{B}(Q, 2 d) \backslash\{P\}} v_{i}(z)=\left(\frac{1}{2}\right)^{N-2} u_{i}(\bar{x})>0 .
$$

From now on, we identify $v_{i}$ with its extension to $P$.

By an elementary computation, $v_{i}$ is seen to satisfy

$$
\left\{\begin{array}{ll}
\Delta v_{i}+\prod_{j=1}^{m} v_{j}^{a_{i j}}=0 & \text { in } B(Q, 2 d) \\
\frac{\partial v_{i}}{\partial \nu}(z)+\frac{N-2}{4 d} v_{i}(z)=-c_{i} \prod_{j=1}^{m} v_{j}(z)^{b_{i j}} & \text { on } \partial B(Q, 2 d) \\
v_{i}(z)>0 & \text { in } \bar{B}(Q, 2 d),
\end{array} \quad \text { for all } i \in J,\right.
$$

where $\nu$ is the outward unit normal vector on the boundary of $B(Q, 2 d)$. Combining the first and third items of (4.10) implies that $v_{i}$ is non-constant in $B(Q, 2 d)$ for all $i \in J$. By a simple maximum-principle argument and since $v_{i}$ is radial about $Q$ we see that $v_{i}$ is strictly decreasing about $Q$ in $B(Q, 2 d)$. Setting $r=|z-Q|$ we have $v_{i}(z)=\psi_{i}(r)$ for some smooth decreasing functions $\psi_{i}:[0,2 d) \rightarrow(0, \infty)$. Using (4.9) and (4.10), these functions are seen to satisfy

$$
\left\{\begin{array}{l}
\psi_{i}^{\prime \prime}(r)+\frac{N-1}{r} \psi_{i}^{\prime}(r)+\prod_{j=1}^{m} \psi_{j}(r)^{a_{i j}}=0 \quad \text { for } 0<r<2 d \\
\psi_{i}^{\prime}(2 d)+\frac{N-2}{4 d} \psi_{i}(2 d)=-c_{i} \prod_{j=1}^{m} \psi_{j}(2 d)^{b_{i j}}
\end{array} \quad \text { for all } i \in J .\right.
$$

By the uniqueness of solutions to this system, there are positive constants $\alpha_{1}, \cdots, \alpha_{m}$ and $\mu$ satisfying

$$
\log \alpha_{i}=\sum_{j=1}^{m} a_{i j} \log \alpha_{j}-\log \left(\mu^{2} N(N-2)\right) \quad \text { for all } i \in J
$$

such that 


$$
\psi_{i}(r)=\frac{\alpha_{i}}{\left(\mu^{2}+r^{2}\right)^{(N-2) / 2}} \quad \text { for all } i \in J .
$$

Using this in equation (4.8) with $z=T y$, we have

$$
\begin{aligned}
u_{i}(y) & =\left(\frac{|T y-P|}{2 d}\right)^{N-2} \frac{\alpha_{i}}{\left(\mu^{2}+|T y-Q|^{2}\right)^{(N-2) / 2}} \\
& =\frac{\beta_{i}}{\left(\sigma^{2}+\left|y-y^{0}\right|^{2}\right)^{(N-2) / 2}}
\end{aligned}
$$

for all $y \in \overline{\mathbb{R}_{+}^{N}}$ and all $i \in J$, where

$$
\beta_{i}=\left(\frac{4 d^{2}}{\mu^{2}+4 d^{2}}\right)^{(N-2) / 2} \alpha_{i}, \quad \sigma^{2}=\mu^{2}\left(\frac{4 d^{2}}{\mu^{2}+4 d^{2}}\right)^{2} \quad \text { and } \quad y^{0}=\bar{x}-d \frac{\mu^{2}-4 d^{2}}{\mu^{2}+4 d^{2}} e_{N} .
$$

By (4.12) and the expressions of $\sigma^{2}$ and $\beta_{i}$, it is routine to verify that $\sigma^{2}$ and $\beta_{1}, \cdots, \beta_{m}$ satisfy (1.6). Moreover, by using both the second item of (4.11) and (4.12) one may verify that (1.10) is satisfied.

\section{APPENDix}

Lemma 5.1. Let $R>0$ and suppose $v$ is a solution of

$$
\begin{cases}-\Delta v \geq 0 & \text { in } B_{R}^{+} \\ \frac{\partial v}{\partial y_{N}}<0 & \text { on }\left(\partial B_{R}^{+} \cap \partial \mathbb{R}_{+}^{N}\right) \backslash\{0\} \\ v>0 & \text { on } \overline{B_{R}^{+}} \backslash\{0\}\end{cases}
$$

Then $v(y) \geq \min _{\partial B_{R} \cap \overline{\mathbb{R}_{+}^{N}}} v$ for all $y \in \overline{B_{R}^{+}} \backslash\{0\}$.

Proof. Set $m_{R}=\min _{\partial B_{R} \cap \overline{\mathbb{R}_{+}^{N}}} v$ and fix $0<\epsilon<R$. Define

$$
\phi(y)=m_{R} \frac{\epsilon^{2-N}-|y|^{2-N}}{\epsilon^{2-N}-R^{2-N}} \quad \text { for } \epsilon \leq|y| \leq R .
$$

One may easily verify that $v-\phi$ satisfies

$$
\begin{cases}-\Delta(v-\phi) \geq 0 & \text { in } B_{R}^{+} \backslash B_{\epsilon} \\ \frac{\partial}{\partial y_{N}}(v-\phi)<0 & \text { on } \partial\left(B_{R}^{+} \backslash B_{\epsilon}\right) \cap \partial \mathbb{R}_{+}^{N} \\ v-\phi \geq 0 & \text { on }\left(\partial B_{R} \cup \partial B_{\epsilon}\right) \cap \mathbb{R}_{+}^{N} .\end{cases}
$$

According to the maximum principle and the third item of (5.1), if $v-\phi$ is negative at any point of $\overline{B_{R}^{+}} \backslash B_{\epsilon}$, then there is $x_{0} \in \partial \mathbb{R}_{+}^{N} \cap\{\epsilon<|y|<R\}$ such that

$$
\underset{\min _{R}^{+} \backslash B_{\epsilon}}{ }(v-\phi)=(v-\phi)\left(x_{0}\right)<0 .
$$

Moreover, since $x_{0} \in \partial \mathbb{R}_{+}^{N}$ is a minimizer of $v-\phi$, we have $\frac{\partial}{\partial y_{N}}(v-\phi)\left(x_{0}\right) \geq 0$. This violates the second item of (5.1). We conclude that $v \geq \phi$ in $\overline{B_{R}^{+}} \backslash B_{\epsilon}$. Finally, if $y \in \overline{B_{R}^{+}} \backslash\{0\}$, and if $0<\epsilon<|y| / 2$ we have 


$$
v(y) \geq m_{R} \frac{\epsilon^{2-N}-|y|^{2-N}}{\epsilon^{2-N}-R^{2-N}} .
$$

Letting $\epsilon \rightarrow 0$ in this inequality gives the desired result.

The proofs of the following two lemmas can be found in [18, [4] or [17].

Lemma 5.2. Let $f \in C^{1}\left(\mathbb{R}_{+}^{N}\right), N \geq 2$ and $b>0$. If $f$ satisfies

$$
f(y) \geq\left(\frac{\lambda}{|y-x|}\right)^{b} f\left(x+\frac{\lambda^{2}(y-x)}{|y-x|^{2}}\right) \quad \text { for all } y \in \mathbb{R}_{+}^{N}, x \in \partial \mathbb{R}_{+}^{N} \text { and } \lambda>0
$$

then $f(y)=f\left(y_{N} e_{N}\right)$ for all $y \in \mathbb{R}_{+}^{N}$, where $e_{N}=(0, \cdots, 0,1)$.

Lemma 5.3. Let $f \in C^{1}\left(\mathbb{R}^{N}\right), N \geq 1$ and $b>0$. Suppose that for every $x \in \mathbb{R}^{N}$, there exists $\lambda(x)>0$ such that

$$
\left(\frac{\lambda(x)}{|y-x|}\right)^{b} f\left(x+\frac{\lambda(x)^{2}(y-x)}{|y-x|^{2}}\right)=f(y) \quad \text { for all } y \in \mathbb{R}^{N} \backslash\{x\} .
$$

Then there exists $a \geq 0, d>0$ and $\bar{x} \in \mathbb{R}^{N}$ such that

$$
f(x)= \pm\left(\frac{a}{d+|\bar{x}-x|^{2}}\right)^{b / 2}
$$

\section{REFERENCES}

[1] T. Aubin. Équations différentielles non linéaires et problìne de Yamabe convernant la courbure scalaire. J. Math. Pures Appl., 55:269-296, 1976.

[2] G. Bianchi. Non-existence of positive solutions to semilinear elliptic equations on $\mathbb{R}^{n}$ or $\mathbb{R}_{+}^{n}$ through the method of moving planes. Comm. Partial Differential Equations, 22:1671-1690, 1997.

[3] L. Caffarelli, B. Gidas, and J. Spruck. Asymptotic symmetry and local behavior of semilinear equations with critical Sobolev growth. Comm. Pure Appl. Math., 42:271-297, 1989.

[4] M. Chipot, I. Shafrir, and M. Fila. On the solutions to some elliptic equations with nonlinear Neumann boundary conditions. Adv. Differential Equations, 1:91-110, 1996.

[5] M. Chipot, I. Shafrir, and G. Wolansky. On the solutions of Liouville systems. J. Differential Equations, 140:59105, 1997.

[6] D. G. De Figueiredo and P. L. Felmer. A Liouville-type theorem for elliptic systems. Ann. Scuola. Norm. Sup. Pisa, 21:387-397, 1994.

[7] K. Deng and H. A. Levine. The role of critical exponents in blow-up theorems: The sequel. J. Math. Anal. App., 243:85-126, 2000.

[8] Z. Djadli, A. Malchiodi, and M. Ould Ahmedou. Prescribing scalar and boundary mean curvature on the three dimensional half sphere. J. Geom. Anal., 13(2):255-289, 2003.

[9] J. Escobar. Conformal deformation of a Riemannian metric to a scalar flat metric with constant mean curvature on the boundary. Ann. of Math., 136:1-50, 1992.

[10] J. Escobar. The Yamabe problem on manifolds with boundary. J. Differential Geom., 35:21-84, 1992.

[11] M. Escobar and M. A. Herero. Boundedness and blow up for a semilinear reaction-diffusion system. J. Differential Equations, 89:176-202, 1991.

[12] Z. Han and Y. Li. The Yamabe problem on manifolds with boundary: Existence and compactness results. Duke Math. J., 99(3):489-542, 1999.

[13] Z. Han and Y. Li. The existence of conformal metrics with constant scalar curvature and constant boundary mean curvature. Comm. Anal. Geom., 8(4):809-869, 2000.

[14] J. M. Lee and T. H. Parker. The Yamabe problem. Bull. Amer. Math. Soc., 17(1):37-91, 1987.

[15] H. A. Levine. The role of critical exponents in blow-up theorems. SIAM Reviews, 32:262-288, 1990.

[16] H. Li and M. Wang. Critical exponents and lower bounds of blow-up rate for a reaction-diffusion system. Nonlinear Analysis, 63:1083-1093, 2005. 
[17] Y. Li and L. Zhang. Liouville-type theorems and Harnack-type inequalities for semilinear elliptic equations. J. Anal. Math, 90:27-87, 2003.

[18] Y. Li and M. Zhu. Uniqueness theorems through the method of moving spheres. Duke Math. J., 80(2):383-417, 1995.

[19] F. Marques. Existence results for the Yamabe problem on manifolds with boundary. Indiana Univ. Math. J., 54:1599-1620, 2005.

[20] E. Mitidieri. A Rellich type identity and applications. Comm. Partial Diff. Equations, 18:125-171, 1993.

[21] E. Mitidieri. Nonexistence of positive solutions of semilinear elliptic systems in $R^{n}$. Differential Integral Equations, 9(3):465-479, 1996.

[22] R. Schoen. Conformal deformation of a Riemannian metric to constant scalar curvature. J. Differential Geom., 20(2):479-495, 1984.

[23] N. Trudinger. Remarks concerning the conformal deformation of Riemannian structures on compact manifolds. Ann. Scuola Norm. Sup. Cl. Sci., 22(3):265-274, 1968.

[24] S. Zheng. Nonexistence of positive solutions to a semilinear elliptic system and blow-up estimates for a reactiondiffusion system. J. Math. Anal. Appl., 232:293-311, 1999.

Department of Mathematics, University of Florida, 358 Little Hall, PO Box 118105, Gainesville FL 32611-8105

E-mail address: mgluck@ufl.edu

E-mail address: leizhang@ufl.edu 